\title{
Theoretical Study of High Near-Field Enhancement Associated with the Lasing Action in Strongly Coupled Plasmonic Nanocavity Arrays
}

\author{
Montacer Dridi,* Amine Jaouadi, Florent Colas, and Chantal Compère
}

Cite This: J. Phys. Chem. C 2021, 125, 749-756

ABSTRACT: Lasing action in strongly coupled plasmonic nanocavity arrays was investigated both theoretically and experimentally. This phenomenon was generated by coupling a gain medium with a surface lattice plasmon resonance mode. In this paper, the near-field properties of this laser are investigated theoretically. It is showed that the lasing action is associated with a strong near-field enhancement at the emission wavelength that can be 4 orders of magnitude higher than what could be generated by the same nanostructure without the gain medium. The field enhancement depends non-linearly on the pumping energy. Finally, the enhancement mechanism in such an active system is assumed to rely mainly on energy transfer at the nanoscale from molecules composing the gain medium to the plasmonic mode.

\section{INTRODUCTION}

Plasmons are resonant collective oscillations of the conduction electrons confined in nanoscale volumes at the interface between a metal (usually noble metal) and dielectric mediums. ${ }^{1}$ They have been the focus of extensive research in the past decades. ${ }^{2-6}$ Their fundamental property is that they can beat the diffraction limit. Light is then concentrated in a volume much smaller than the wavelength, leading to a strong enhancement of the electromagnetic field. Recent studies showed that the interaction of a gain medium with plasmons can yield to the lasing effect. ${ }^{7-10}$ This nanoscale phenomenon can occur when the quantum emitters-composing the gain medium-located at the vicinity of metallic nanostructures undergo an inversion of population that couples to a plasmon mode. In the near-field region, the lasing effect leads to energy transfer, amplification of both spontaneous and stimulated emission, and ultra-fast dynamics. In the far-field region, it yields to a coherent emission of photons with a narrow linewidth (few nanometers). This only occurs when the pumping energy goes beyond a threshold. ${ }^{11-19}$ According to the literature, two different classes of plasmon-based lasers have been reported. They are distinguished from the nature of the quantum emitters integrated into the so-called gain medium. The first class uses semiconductors, ${ }^{20-28}$ while the second one uses fluorescent molecules. ${ }^{11,15,16,29-32}$ Different geometries have been reported, such as silver film, ${ }^{25}$ gold film, ${ }^{33}$ nanowire, ${ }^{34}$ nanohole array, ${ }^{20}$ bowtie array, ${ }^{29}$ and nanocylinder array. $30,32,35$

In this paper, we investigate the lasing action in a periodic array of metallic nanoparticles embedded in a molecular gain medium under optical pumping, which will be referred to us hereafter as the active structure. The use of such a system as a plasmon-based laser has been demonstrated both theoretically and experimentally by the groups of Odom and Schatz. ${ }^{30}$ Experimentally, the far-field properties of the lasing system were demonstrated; more precisely, the spatial coherence (directional beam emission) and picosecond dynamics of relaxation were shown. The role of the surface lattice plasmon mode providing the necessary feedback was also shown. Theoretically, the model developed was based on a semiquantum electromagnetic approach. The simulations dealt only with the active optical responses in the far field and the calculation of the gain. The current work completes those studies by focusing on the near-field properties resulting from the lasing action. First, we raise the problem and the difficulty of defining the enhancement in the active system as the resonance in such a system tune away from the incident field wavelength (which is with a very narrow spectral bandwidth). Then, the near-field enhancement below and above the threshold is investigated. They are both compared with the near-field generated by the nanostructure without the gain medium, which will be called the passive structure hereafter. Note that such a comparison cannot be predicted due to the intrinsic feedback between the nanoparticles and molecule within the gain. Indeed, in the active system, the optical properties depend strongly on the pumping energy as well as

Received: September 23, 2020

Revised: December 8, 2020

Published: December 22, 2020 
on the coupling strength between molecules in the gain and the plasmonic field. Finally, in this paper, we discuss the mechanism behind that enhancement by investigating the dynamic of the stimulated emission process. Such a theoretical advancement would further move towards the understanding and application of the near-field properties in a plasmon-based laser. Those properties could lead to a new type of surfaceemitting laser able to generate a highly confined electric field.

\section{METHODS}

2.1. Description of the Active and the Passive Structures. The system of interest was reported recently in refs 30,32 . The active structure is composed of a twodimensional (2D) array of gold nanocylinders (NC) deposited on a glass substrate and covered by a gain layer made up by IR140 dye molecules embedded in polyurethane (Figure 1). The

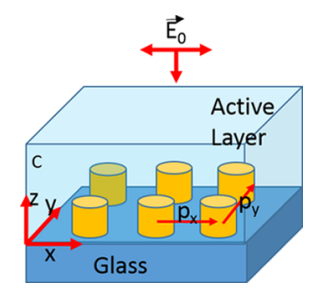

Figure 1. Scheme of the periodic nanocavity array laser.

diameter of the NC is $d=130 \mathrm{~nm}$, the height $h=60 \mathrm{~nm}$, and the $2 \mathrm{D}$-array periodicity $p_{x}=p_{y}=600 \mathrm{~nm}$. The dye molecules absorb at $\lambda_{\mathrm{a}}=800 \mathrm{~nm}$ and emit at $\lambda_{\mathrm{e}}=870 \mathrm{~nm}$. The concentration of IR-140 in the polyurethane layer was $2 \times 10^{18}$ $\mathrm{cm}^{3}$. The system is optically pumped by an $800 \mathrm{~nm}$ femtosecond-pulsed laser $E_{0}(t)$ at normal incidence. For such a plasmon-based nanolaser, a directional beam emission associated with plasmon excitation and energy transfer were demonstrated in ref 30 .

The passive structure is the same as the active one except that there is no dye molecule in the polymer layer. Their properties are calculated by finite-difference time-domain (FDTD) calculation.

2.2. Semi-Quantum Approach for Lasing Modeling. The lasing system is composed by molecules, metallic nanoparticles, and an electromagnetic source. The electromagnetic fields of such a system can be simulated with the semi-quantum approach presented in previous studies. $50,30,31$ We expose briefly this approach hereafter. Molecules will be described by quantum mechanics and considered as a fourenergy-level system (Figure 2). The electromagnetic source and the metallic nanoparticle with its dispersive properties are described classically by Maxwell equations.

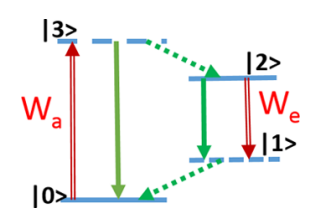

Figure 2. Schematic representation of transitions of the molecule as a four-energy-level system. Single (double) arrows are for spontaneous transition (stimulated). $W_{\mathrm{a}}$ and $W_{\mathrm{e}}$ describe the simulated absorption and the simulated emission rates, expressed in molecule per $\mathrm{m}^{3}$ per second. Single continuous (dashed) arrows are for slow (fast) spontaneous transitions.
When an electromagnetic source at optical frequencies irradiates a set of molecules assumed initially at the ground state $\mid 0>$, a displacement of the electron cloud is induced, which leads to microscopic molecular polarization and a change of the population density of the quantum states. The molecular polarization generates a current source that modifies the total electromagnetic field in the system.

To describe this interaction between the molecules and electromagnetic fields, a set of nonlinear coupled partial differential equations was proposed. ${ }^{5,36-39}$ In this work, we briefly review those equations for consistency purposes. Details about numerical implementation can be found in ref 5. First, the dynamic of the density of each quantum state is described using the rate equation model. Each population density is governed by absorption and emission rates. For a molecule considered as a four-energy-level system (see Figure 2), the time evolution of population densities are described by the following equations:

$$
\left\{\begin{array}{l}
\dot{\rho}_{0}=\frac{\rho_{1}(t)}{\tau_{10}}+\frac{\rho_{3}(t)}{\tau_{30}}-W_{\mathrm{a}} \\
\dot{\rho}_{1}=\frac{\rho_{2}(t)}{\tau_{21}}-\frac{\rho_{1}(t)}{\tau_{10}}-W_{\mathrm{e}} \\
\dot{\rho}_{2}=\frac{\rho_{3}(t)}{\tau_{32}}-\frac{\rho_{2}(t)}{\tau_{21}}+W_{\mathrm{e}} \\
\dot{\rho}_{3}=-\frac{\rho_{3}(t)}{\tau_{32}}-\frac{\rho_{3}(t)}{\tau_{30}}+W_{\mathrm{a}}
\end{array}\right.
$$

where

$$
\left\{\begin{array}{l}
W_{\mathrm{a}}=\frac{1}{\hbar \omega_{30}} \vec{E}(t) \cdot \frac{\mathrm{d} \vec{P}_{30}(t)}{\mathrm{d} t} \\
W_{\mathrm{e}}=\frac{1}{\hbar \omega_{21}} \vec{E}(t) \cdot \frac{\mathrm{d} \vec{P}_{21}(t)}{\mathrm{d} t}
\end{array}\right.
$$

The terms $\frac{\vec{E} \cdot \mathrm{d} \vec{P}_{30}}{\mathrm{~d} t}$ and $\frac{\vec{E} \cdot \mathrm{d} \vec{P}_{21}}{\mathrm{~d} t}$ are the classical expressions for stimulated energy transfer between an electromagnetic wave and the molecule. $W_{\mathrm{a}}$ and $W_{\mathrm{e}}$ describe respectively the stimulated absorption process and stimulated emission process. Both are expressed in molecule per $\mathrm{m}^{3}$ per second. Note that $W_{\mathrm{a}}$ and $W_{\mathrm{e}}$ depend on the total electric field $\vec{E}(t)$ in the system including the nanoparticle.

The time evolution of population density $\rho_{i}$ is governed also by a spontaneous emission process with $1 / \tau_{i, j}$ is the spontaneous decay rate (expressed in $\mathrm{s}^{-1}$ ).

In the set of eqs 1 and 2 above, we have neglected the polarization resulting from transitions from state $\mid 3>$ to $\mid 2>$ and from state $\mid 1>$ to $\mid 0>$, presented by a green dashed line in Figure 2 , as they are considered very fast with a low energy.

Second, the polarization $\vec{P}_{i, j}$ resulting from each optically pumped transition will be calculated by solving the following equation: ${ }^{37}$

$$
\frac{\mathrm{d}^{2} \vec{P}_{i, j}(t)}{\mathrm{d} t^{2}}+\Delta \omega_{i, j} \frac{\mathrm{d} \vec{P}_{i, j}}{\mathrm{~d} t}+\omega_{i, j}^{2} \vec{P}_{i, j}=\kappa_{i, j} \rho_{i, j}(t) \vec{E}(t)
$$

Hence, we have a system of two equations: the first one for the couple $(i=3, j=0)$ and the second one for the couple $(i=$ 

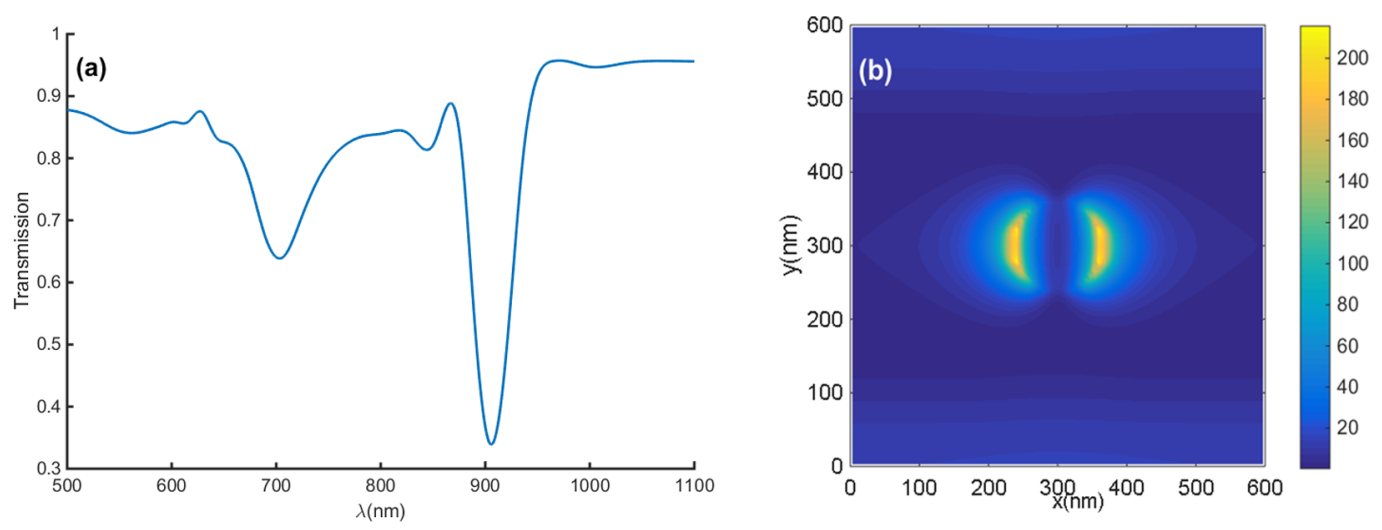

Figure 3. Optical properties of the passive structure. (a) Transmission spectra featuring a narrow lattice plasmon resonance at $\lambda_{\mathrm{r}}=905 \mathrm{~nm}$ and a broad resonance at $\lambda=720 \mathrm{~nm}$. (b) Enhancement factor $F_{\mathrm{p}}$ at $\lambda_{\mathrm{r}}=905 \mathrm{~nm}$.

$2, j=1)$. Where $\Delta \omega_{i, j}$ is the bandwidth of the transition of interest, $\kappa_{i, j}=6 \pi \varepsilon_{0} c^{3} \tau_{i, j} / \omega_{i, j}^{2}$ and $\rho_{i, j}(t)=\rho_{j}(t)-\rho_{i}(t)$ are the population density difference between the upper and lower states.

Finally, the current source $\vec{J}=\frac{\partial \vec{P}_{21}}{\partial t}+\frac{\partial \vec{P}_{30}}{\partial t}$ is injected in the Maxwell equations to take into account the modification of the total field due to the change of the densities $\rho(t)$ and $\vec{P}$ :

$$
\left\{\begin{array}{l}
\nabla \times \vec{E}(t)=-\mu_{0} \frac{\partial \vec{H}(t)}{\partial t} \\
\nabla \times \vec{H}(t)=\varepsilon \frac{\partial \vec{E}(t)}{\partial t}+\vec{J}
\end{array}\right.
$$

The above set of coupled equations (eqs 1-4) is selfconsistent and describes both the linear and nonlinear regimes.

For the numerical simulation, we assumed that the spontaneous decay rate are $\tau_{21}=\tau_{30}=1 \mathrm{~ns}$ and $\tau_{32}=\tau_{10}=$ 10 fs. The spectral bandwidth of the absorption (transition: 31) and emission (transition: 2-1) are $\Delta \lambda_{\mathrm{a}}=\Delta \lambda_{\mathrm{e}}=70 \mathrm{~nm}$. The absorption wavelength is $\lambda_{\mathrm{a}}=800 \mathrm{~nm}$, and the emission wavelength is $\lambda_{\mathrm{e}}=870 \mathrm{~nm}$.

2.3. FDTD Simulations of the Active Structure. A timestepping algorithm is developed to solve eqs $1-4$ in threedimensional FDTD (finite-difference time-domain) code. The computational volume is truncated in the $z$ direction using the convolutional perfectly matched layer technique. ${ }^{40}$ In the $x$ and $y$ directions, periodic boundary conditions are implemented. The stability and convergence criteria are similar to the traditional FDTD algorithm. ${ }^{39}$ To introduce the source on the computational volume, the total-field/scattered-field formulation was used. The mesh discretization is $\Delta x=\Delta y=\Delta z=5$ $\mathrm{nm}$. The courant stability factor is $C_{\mathrm{s}}=0.9$.

The glass substrate and polymer refractive indices are both taken to be equal to 1.5. The gold permittivity is described using the Drude-Lorentz model. ${ }^{41}$

\section{RESULTS AND DISCUSSION}

Figure 3 shows the properties of the passive structure. The transmission spectra (Figure 3a) presents a broad peak and a narrow peak at $720 \mathrm{~nm}$ and $\lambda_{\mathrm{r}}=905 \mathrm{~nm}$, respectively. The former corresponds to the resonance of an isolated nanoparticle, while the latter to a band-edge lattice plasmon mode $^{42-45}$ (surface lattice plasmon resonance, SLPR). Figure
$3 b$ shows the enhancement factor $F_{p}\left(\lambda_{\mathrm{r}}\right)$ for the passive structure defined by

$$
F_{\mathrm{p}}\left(\lambda_{\mathrm{r}}\right)=\left|\frac{E\left(\lambda_{\mathrm{r}}\right)}{E_{0}\left(\lambda_{\mathrm{r}}\right)}\right|^{2}
$$

where $E\left(\lambda_{\mathrm{r}}\right)$ is the total field and $E_{0}\left(\lambda_{\mathrm{r}}\right)$ is the field without nanoparticle at the wavelength $\lambda_{\mathrm{r}}$.

$F_{\mathrm{p}}\left(\lambda_{\mathrm{r}}\right)$ is calculated in the $x-y$ plane parallel to the substrate at a distance of $10 \mathrm{~nm}$ from the nanoparticle top face at the resonance wavelength. Figure $3 \mathrm{~b}$ shows a strong optical field concentrated on the edges of the individual gold nanoparticles. The surface plasmon resonance generates a near-field enhancement of about 200 at $\lambda_{\mathrm{r}}=905 \mathrm{~nm}$. For the calculation of $F_{\mathrm{p}}\left(\lambda_{\mathrm{r}}\right)$, the incident source $E_{0}$ is a pulse centered at an arbitrary frequency with a very large spectral bandwidth and assumed to be spatially homogeneous. The polarization is parallel to the $x$ axis. The spectrum of the incident source for the passive structure is represented by a blue dashed line in Figure 4. We also plot the incident source for the active structure for comparison purposes.

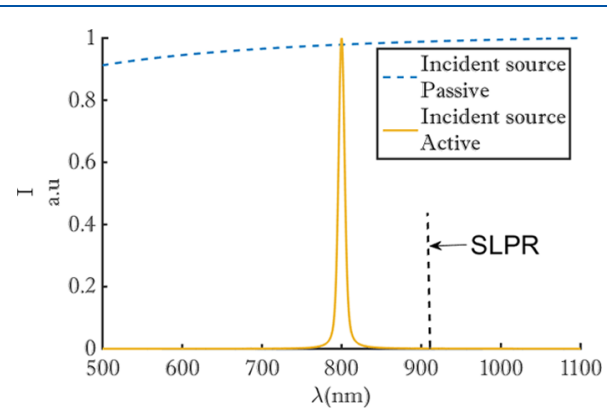

Figure 4. Incident source conditions in the frequency domain: The active structure is excited by a very narrow pulse centered at $\lambda=800$ $\mathrm{nm}$. The passive structure is excited by a very broad pulse. The vertical dark dashed line indicates the surface lattice plasmon resonance.

3.1. Near-Field Enhancement in the Active Structure. Now, let us focus on the optical response of the active system where the dye molecules are considered in the fundamental state at $t=0$ (fluctuations are neglected). An incident light pulse centered at $\lambda_{\mathrm{a}}=800 \mathrm{~nm}$ pumps the system (Figure 4). The output field (emission) of the whole system is calculated far from the nanoparticle at an off-angle. The far-field spectrum 
is shown in Figure 5. The lasing action produces a narrow emission peak of $3 \mathrm{~nm}$ bandwidth at $\lambda_{\mathrm{r}}=905 \mathrm{~nm}$. The inset of

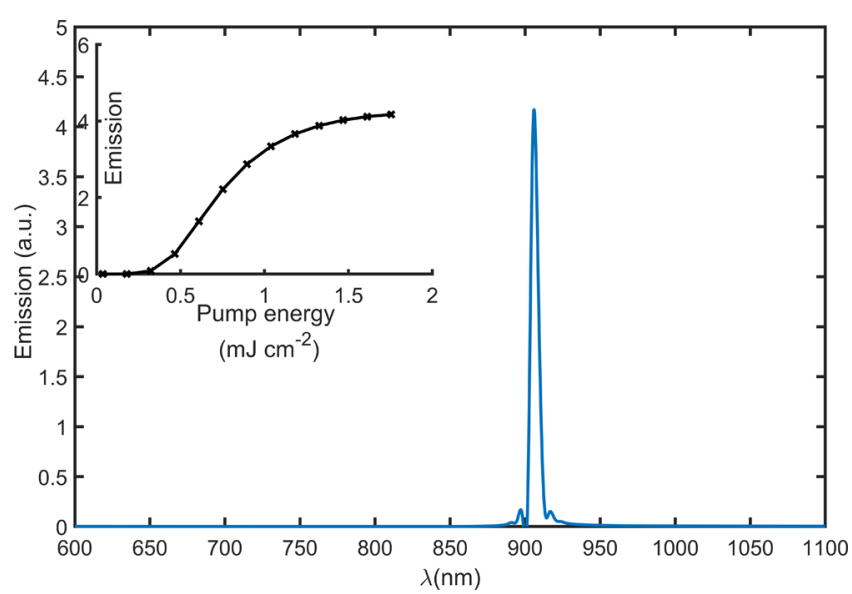

Figure 5. Far-field spectrum of the active structure pumped by a 1.5 $\mathrm{mJ} \cdot \mathrm{cm}^{-2}$. Inset: Output (the emission) vs input energy (the pumping).

Figure 5 presents the output vs input energy (pumping energy). The emission occurs for a pump energy above the threshold and is estimated to be $0.5 \mathrm{~mJ} \cdot \mathrm{cm}^{-2}$ in this case. ${ }^{30}$

Those far-field properties were demonstrated experimentally in refs 30 and 32 . We pursue here those studies by focusing on the near-field properties. For that, we will be providing a new definition to the enhancement that will provide a comparison between a passive and an active structure. Then, we will suggest what kind of mechanism is behind the enhancement. One should note that the near-field enhancement in the active system depends strongly on a variety of quantum and classical parameters. Indeed, and unlike the passive structure, the optical response of an active structure in the near-field region depends strongly on the pumping energy as the pumping energy will modify the molecular polarization and the population density of each molecular state. This will modify the total field present in the system and thus can result in new optical and plasmonic properties. In other words, the near field in the active system will depend strongly on the gain (inversion) close to the nanoparticles, on the losses in the metal structure, and on the coupling strength between the molecule and plasmon. In addition, the coupling strength itself depends on a variety of parameters among them: the emission cross section of the dye molecule, the amount of gain achieved close to the plasmonic structure, the overlap between the plasmonic resonance wavelength and the emission of the molecule, the ratio of spontaneous emission versus stimulated emission, etc. All those parameters make the near-field enhancement unpredictable. Investigating these parameters is of great importance both from the fundamental and applied sides.

Now, let us focus on the calculation of the near-field enhancement associated with the demonstrated far-field emission. In Figure 6, we calculate the enhancement factor as a function of the pumping energy at the vicinity of the nanoparticle. The enhancement factor for the active structure $\left(F_{\mathrm{a}}\left(\lambda_{\mathrm{r}}\right)=\left|\frac{E\left(\lambda_{\mathrm{r}}\right)}{E_{0}\left(\lambda_{\mathrm{r}}\right)}\right|^{2}\right)$ was calculated at $\lambda_{\mathrm{r}}=905 \mathrm{~nm}$ in the semilogarithmic scale for different pumping energies and is shown in Figure 6a. For very weak pumping energies, there is no significant enhancement compared to the passive structure whose enhancement $F_{\mathrm{p}}\left(\lambda_{\mathrm{r}}\right)$ is represented by a green horizontal dashed line. The enhancement grows rapidly and nonlinearly with a pumping energy reaching $10^{5}$ at the threshold. Then, it still increases but more slowly to $10^{6}$ at a pumping energy of $1.2 \mathrm{~mJ} \cdot \mathrm{cm}^{-2}$. This result shows that the enhancement factor strongly depends on the incident pumping energy. The maximum of the near-field enhancement is reached above the threshold. However, even below the threshold, the coupling between the quantum emitters of the gain material and the plasmon mode enhances strongly the near field. The field map above the threshold at $\lambda_{\mathrm{r}}=905 \mathrm{~nm}$ is plotted in Figure $6 \mathrm{~b}$. The highest enhancement is observed close to the NC, but a significant enhancement is achieved also in locations far from the nanoparticle. For example, an enhancement up to 30,000 is reached at more than $150 \mathrm{~nm}$ away from the nanoparticle edge along the $x$ axis.

Note that, for the calculation of $F_{\mathrm{a}}\left(\lambda_{\mathrm{r}}\right)$, the incident source $E_{0}$ (Figure 4 ) is a pulse with a very narrow spectral bandwidth (few nanometers) and centered at the frequency of the absorption transition of the gain molecule $(800 \mathrm{~nm})$. In the section below, we will be further comparing the enhancement factor in both active and passive structures.

3.2. Mechanism of the Near-Field Enhancement in an Active System. Near-field enhancement is one of the most remarkable phenomena associated with plasmons. For a passive structure, the incident light at the resonance frequency of the nanostructure interacts strongly with the free electrons of the conduction band of the metal generating a quasi-particle called plasmon polariton. This electron-photon duality strengthens significantly the local electric fields within the
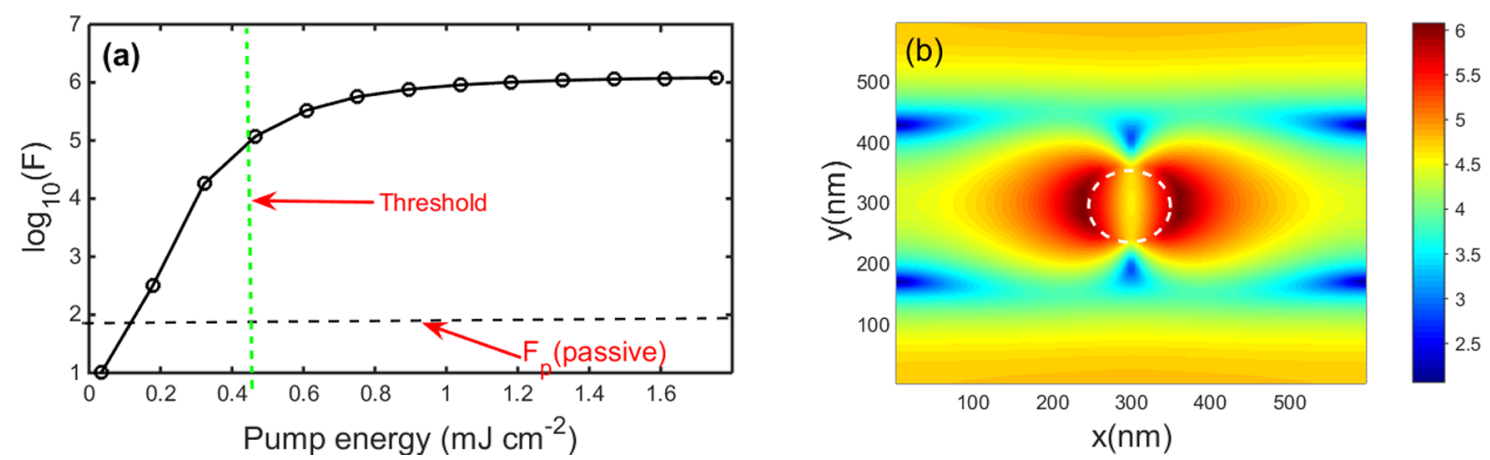

Figure 6. Optical properties of the active structure at the nanoscale. (a) Enhancement factor $F_{a}$ as the function of the pump energy. (b) Field map above the threshold of lasing in the logarithmic scale. 
metal nanostructures. For instance, the passive structure studied here enhances the local field 200 times with respect to the incident field (see Figure $3 b$ ). In the case of the active structure, the incident light is assumed to interact poorly with the nanoparticle as the incident light (i.e., the pump pulse) tunes away from the resonance of the passive structure. Indeed, the incident light in the active system is centered at $\lambda_{\mathrm{a}} \approx 800$ $\mathrm{nm}$, which is the absorption wavelength of the dye molecule. In Figure 7 , we superimpose the incident field, the absorption,

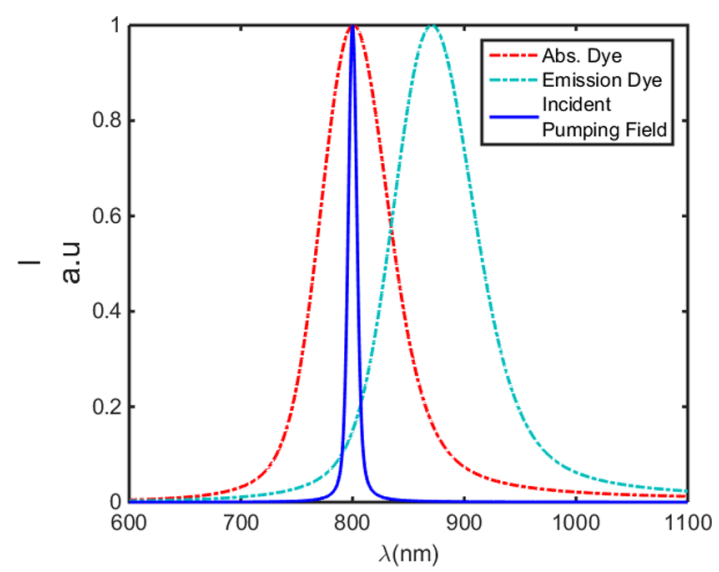

Figure 7. Schematic description of spectral overlaps between the pumping incident field, the absorption, and the emission of the dye.

and the emission spectra of the dye. We observe that there is no significant component of the incident pulse at the resonance frequency of the nanostructure. Thus, the direct interaction between the incident light (pumping) and nanoparticle could be assumed to be very weak and could not be responsible of the observed enhancement.

One could conclude that the enhancement mechanism of the local field in the active structure is rather assumed to originate from the strong coupling between the molecular polarization state $\vec{P}_{21}$ (emission transition) and the total field in the system. Strong coupling means energy transfer between molecules and the plasmonic structure that is possible only for high pumping above the threshold. The energy delivered by the gain (inversion) is captured by the nanoparticle leading to an enhancement of the field present in the system. The energy transfer from the population in state $\mid 2>$ to state $\mid 1>$ can be calculated by eq 2 at an instant time $t$. We define $S$ as being the time-averaged quantity of $W_{\mathrm{e}}=\frac{1}{\hbar \omega_{21}} \cdot \frac{\vec{E} \cdot \mathrm{d} \overrightarrow{2}_{21}}{\mathrm{~d} t}$, so $S=\frac{1}{n} \sum_{i=1}^{n} W_{\mathrm{e}}\left(t_{i}\right)$ with $t_{i}=i \cdot \Delta t$ ( $\Delta t$ is the temporal discretization) and $n \cdot \Delta t$ is the propagation time of the incident pulse. We find that, in all locations around, nanoparticle $S$ has negative values, meaning that the population of the upper state 2 is decreasing by a stimulated process according to eq $1 . S$ depends on the spatial coordinates and is proportional to the time-averaged population density of the quantum state $12>\left(\rho_{2}\right)$ that relaxes to state $11>$ through a stimulated emission process. The unit of $S$ is molecule per $\mathrm{m}^{3}$ per second (molecule $/ \mathrm{m}^{3} \cdot \mathrm{s}$ ). We could write $S$ as

$$
S=-\gamma_{x, y}^{\text {stim }} \frac{\sum_{i=1}^{n} \rho_{2}\left(t_{i}\right)}{n}
$$

where $\gamma_{x, y}^{\text {stim }}$ will represent the stimulated emission rate expressed in $\mathrm{s}^{-1}$.
Calculation of $\gamma_{x, y}^{\text {stim }}$ is presented in Figure 8 in a plane parallel to the substrate and at a distance of $10 \mathrm{~nm}$ from the

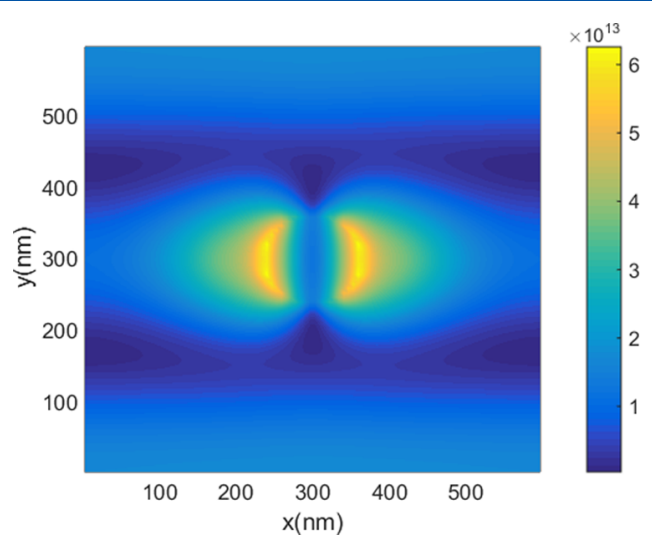

Figure 8. Stimulated emission rate $\gamma^{\text {stim }}$ calculated in a plane $(x-y)$ separated by $10 \mathrm{~nm}$ from the nanoparticle top surface above the threshold of lasing.

nanoparticle top surface. We retrieve the picosecond dynamics for the stimulated emission rate $\gamma_{x, y}^{\text {stim }}$ as demonstrated experimentally in ref 32. Especially, at locations very close to the nanoparticle, $\gamma_{x, y}^{\text {stim }}$ is higher, suggesting that the energy flows from the gain molecule to the nanoparticle. Most importantly, the spatial distribution of $\gamma_{x, y}^{\text {stim }}$ matches very well with the field enhancement map represented in Figure $6 \mathrm{~b}$, demonstrating again that the enhancement factor calculated in Figure 6 is indeed associated with the stimulated emission process and energy transfer at the nanoscale.

3.3. Further Comparison between the Near-Field Intensity in the Passive and Active Structures. The nearfield enhancements at $\lambda_{\mathrm{r}}$ of the active and the passive structure were compared on the basis of the usual enhancement factor considering the ratio of the near field at the same wavelength. However, as mentioned previously, in the case of an active structure, the incident field has no significant component at $\lambda_{\mathrm{r}}$. The calculation of $F_{\mathrm{a}}$ at $\lambda_{\mathrm{r}}=905 \mathrm{~nm}$ using $F_{\mathrm{a}}=\left|\frac{E}{E_{0}}\right|^{2}$ does not fully describe the system. For a better comparison between passive and active structures, we decided to compare the nearfield intensity of the active $\left(|E|_{\mathrm{a}}^{2}\left(\lambda_{\mathrm{r}}\right)\right)$ and the passive $\left(|E|_{\mathrm{p}}^{2}\left(\lambda_{\mathrm{r}}\right)\right)$ structures at $905 \mathrm{~nm}$ when excited by the same total fluence, which means to consider all the incoming wavelengths. Indeed, the active system is excited by a pump pulse centered at the absorption wavelength of the dye $(800 \mathrm{~nm})$ with a very narrow spectral bandwidth of few nanometers. The passive structure is excited by a pulse with a much broader spectral bandwidth (blue dashed line, see Figure 4). The power or fluence is referred to us as: fluence $=\frac{1}{T} \int_{0}^{T}\left|E_{0}(t)\right|^{2} \mathrm{~d} t$, with $T$ being the propagation time of the pulse (very long for the active structure and short for the passive). In the context of FDTD simulations, typically $2^{13}$ temporal iterations are required for the passive structure and $2^{17}$ for the active one.

By exciting the passive and active structure such that both receive the same fluence, we could have then a direct comparison between the near field generated at the nanoscale in each structure. Our goal is to determine which type of structure (passive or active) generates the highest near field. Such a comparison is not predictable; in fact, as stated before, 

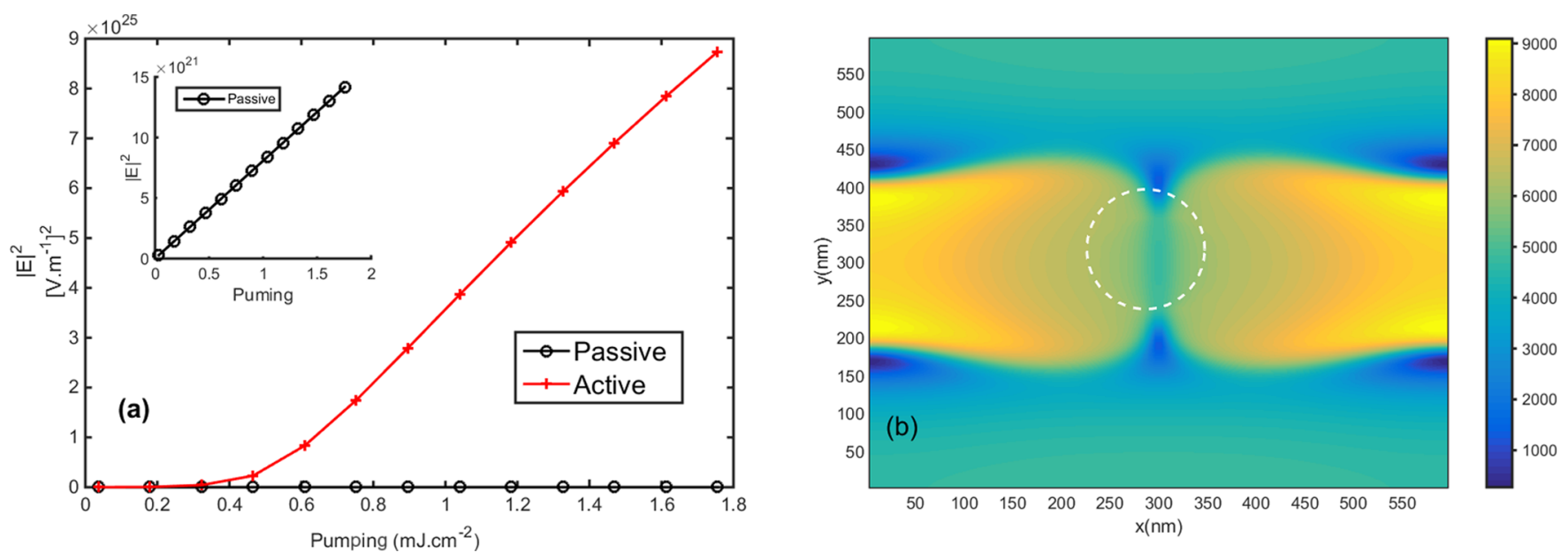

Figure 9. Comparison between the near-field intensity in the active and passive structure. (a) Near-field intensity close to the nanoparticle for different pumping energies, showing a nonlinear behavior for the active and a linear one for the passive (inset). (b) Map of $\frac{\left|E^{2}\right|_{a}}{\left|E^{2}\right|_{p}}$ : As compared to the passive structure, the active structure exhibits a much higher enhancement of the electromagnetic field close and far from the NC.

the narrow band illumination in the active system does not interact directly with the plasmonic system. Instead, it is used to pump efficiently the gain medium. However, pumping the gain medium does not guarantee at all a resonant energy transfer to the plasmon.

Figure 9a shows the field intensity in the frequency domain I $\left.E\left(\lambda_{\mathrm{r}}\right)\right|^{2}$ for different pumping energies (fluence) calculated at a location close to the nanoparticle. The near-field intensity of the active structure increases linearly with the pump power above the threshold. The inset of Figure 9a represents the field intensity in the passive structure. As expected, the intensity increases linearly with pump energy. The intensity reached at the vicinity of the active structure is 4 orders of magnitude higher than with a passive structure receiving the same incident fluence. This demonstrates that, above the threshold, the near field produced in the active structure is much higher compared to the passive structure, suggesting that such an enhancement is due to the coupling between the gain molecules and the plasmon mode.

In the case of an active system where the incident pulse is at the pump frequency, but the resonance is at the lattice plasmon wavelength, it may be more relevant to express the enhancement of the field with respect to a passive structure receiving the same fluence rather than with respect to the incident field.

Pursuing the comparison between the passive and active structure, Figure $9 \mathrm{~b}$ compares the spatial distributions of the near field in the active and the passive structure by mapping the ratio of $\frac{\mid E\left(\left.\lambda_{\mathrm{r}}\right|_{\mathrm{a}} ^{2}\right)}{\mid E\left(\lambda_{\mathrm{r}}\right)_{\mathrm{p}}^{2}}$ at a pump energy above the threshold fixed to $1.8 \mathrm{~mJ} \cdot \mathrm{cm}^{-2}$. Surprisingly, the near field in the active structure is enhanced even at locations far from the $\mathrm{NC}$ as compared to the passive one.

In conclusion, the coupling of a gain medium like dye molecules (such as Rhodamine 6G) with plasmonic mode could be a powerful tool to enhance the local field intensity. The near field generated at the nanoscale in the active structure is 4 orders of magnitude higher than the passive structure. Most importantly, in the case of an SLPR mode, the near-field enhancement in the active system is more delocalized and extends to several hundreds of nanometers away from the NC. This open very interesting prospects for sensing applications as the sensing volume, meaning the volume in which we can detect the targeted molecule, is much larger in the active structure than in the passive one.

\section{CONCLUSIONS}

This paper demonstrates theoretically that lasing action in a strongly coupled nanoparticle cavity is associated with a high near field that is 4 order of magnitude higher than in a passive structure. Unlike the passive structure, the near-field enhancement increases nonlinearly with the incident energy and extends far beyond the near-field decay length of the individual nanoparticle. We demonstrated that the enhancement of the near field originated from the coupling of the plasmon mode to the molecule through energy transfer at the nanoscale.

Strongly coupled nanocavities arrays sustaining a surface lattice plasmon resonance mode and interacting with quantum emitters may constitute a novel class of plasmonic devices delivering an intense optical signal. The application of such a device ranges from molecular or atomic fingerprinting gas sensing, spectroscopic applications, to efficient photon extraction for solid-state lightening.

\section{AUTHOR INFORMATION}

\section{Corresponding Author}

Montacer Dridi - Ifremer, REM/RDT/LDCM, F-29280

Plouzané, France; ○ orcid.org/0000-0002-2805-1585;

Email: dmontacer@gmail.com

\section{Authors}

Amine Jaouadi - ECE Paris, INSEEC U, Paris 75015,

France; (1) orcid.org/0000-0001-8155-8011

Florent Colas - Ifremer, REM/RDT/LDCM, F-29280

Plouzané, France

Chantal Compère - Ifremer, REM/RDT/LDCM, F-29280 Plouzané, France

Complete contact information is available at:

https://pubs.acs.org/10.1021/acs.jpcc.0c08669

\section{Notes}

The authors declare no competing financial interest. 


\section{ACKNOWLEDGMENTS}

M.D. thanks the Department of Scientific Research at the Ifremer and the region of Brittany for financial support. M.D. thanks O. Abidi for valuable comments. The authors thank M. L. de la Chapelle and G. Schatz for valuable comments.

\section{REFERENCES}

(1) Maier, S. A.; Atwater, H. A. Plasmonics: Localization and guiding of electromagnetic energy in metal/dielectric structures. J. Appl. Phys. 2005, 98, No. 011101.

(2) Odom, T. W.; Schatz, G. C. Introduction to plasmonics. Chem. Rev. 2011, 111, 3667-3668.

(3) Ross, M. B.; Mirkin, C. A.; Schatz, G. C. Optical Properties of One-, Two-, and Three-Dimensional Arrays of Plasmonic Nanostructures. J. Phys. Chem. C 2016, 120, 816-830.

(4) Townsend, E.; Bryant, G. W. Plasmonic Properties of Metallic Nanoparticles: The Effects of Size Quantization. Nano Lett. 2012, 12, 429-434.

(5) Dridi, M.; Schatz, G. C. Model for describing plasmon-enhanced lasers that combines rate equations with finite-difference timedomain. J. Opt. Soc. Am. B 2013, 30, 2791-2797.

(6) Dridi, M.; Vial, A. Modeling of metallic nanostructures embedded in liquid crystals: application to the tuning of their plasmon resonance. Opt. Lett. 2009, 34, 2652-2654.

(7) Stockman, M. I. Spasers Explained. Nat. Photonics 2008, 2, 327329.

(8) Zheludev, N. I.; Prosvirnin, S. L.; Papasimakis, N.; Fedotov, V. A. Lasing Spaser. Nat. Photonics 2008, 2, 351-354.

(9) Bergman, D. J.; Stockman, M. I. Surface Plasmon Amplification by Stimulated Emission of Radiation: Quantum Generation of Coherent Surface Plasmons in Nanosystems. Phys. Rev. Lett. 2003, 90, No. 027402.

(10) Oulton, R. F. Surface plasmon lasers: sources of nanoscopic light. Mater. Today 2012, 15, 26-34.

(11) Deeb, C.; Guo, Z.; Yang, A.; Huang, L.; Odom, T. W. Correlating Nanoscopic Energy Transfer and Far-Field Emission to Unravel Lasing Dynamics in Plasmonic Nanocavity Arrays. Nano Lett. 2018, 18, 1454-1459.

(12) Shahbazyan, T. V. Mode Volume, Energy Transfer, and Spaser Threshold in Plasmonic Systems with Gain. ACS Photonics 2017, 4, 1003-1008.

(13) Hakala, T. K.; Rekola, H. T.; Väkeväinen, A. I.; Martikainen, J.P.; Nečada, M.; Moilanen, A. J.; Törmä, P. Lasing in dark and bright modes of a finite-sized plasmonic lattice. Nat. Commun. 2017, 8, 13687.

(14) Schokker, A. H.; van Riggelen, F.; Hadad, Y.; Alù, A.; Koenderink, A. F. Systematic study of the hybrid plasmonic-photonic band structure underlying lasing action of diffractive plasmon particle lattices. Phys. Rev. B 2017, 95, No. 085409.

(15) Hoang, T. B.; Akselrod, G. M.; Yang, A.; Odom, T. W.; Mikkelsen, M. H. Millimeter-Scale Spatial Coherence from a Plasmon Laser. Nano Lett. 2017, 17, 6690-6695.

(16) Wang, D.; Yang, A.; Wang, W.; Hua, Y.; Schaller, R. D.; Schatz, G. C.; Odom, T. W. Band-edge engineering for controlled multimodal nanolasing in plasmonic superlattices. Nat. Nanotechnol. 2017, 12, 889-894.

(17) Deeb, C.; Pelouard, J.-L. Plasmon lasers: coherent nanoscopic light sources. Phys. Chem. Chem. Phys. 2017, 19, 29731-29741.

(18) Röder, R.; Sidiropoulos, T. P. H.; Tessarek, C.; Christiansen, S.; Oulton, R. F.; Ronning, C. Ultrafast Dynamics of Lasing Semiconductor Nanowires. Nano Lett. 2015, 15, 4637-4643. PMID: 26086355

(19) Samuel, I. D. W.; Namdas, E. B.; Turnbull, G. A. How to recognize lasing. Nat. Photonics 2009, 3, 546-549.

(20) van Beijnum, F.; van Veldhoven, P. J.; Geluk, E. J.; de Dood, M. J. A.; Hooft, G. W.; van Exter, M. P. Surface Plasmon Lasing Observed in Metal Hole Arrays. Phys. Rev. Lett. 2013, 110, 206802.
(21) Sidiropoulos, T. P. H.; Röder, R.; Geburt, S.; Hess, O.; Maier, S. A.; Ronning, C.; Oulton, R. F. Ultrafast plasmonic nanowire lasers near the surface plasmon frequency. Nat. Phys. 2014, 10, 870-876.

(22) Zhang, Q.; Li, G.; Liu, X.; Qian, F.; Li, Y.; Sum, T. C.; Lieber, C. M.; Xiong, Q. A room temperature low-threshold ultraviolet plasmonic nanolaser. Nat. Commun. 2014, 5, 1.

(23) Lu, Y.-J.; Kim, J.; Chen, H.-Y.; Wu, C.; Dabidian, N.; Sanders, C. E.; Wang, C.-Y.; Lu, M.-Y.; Li, B.-H.; Qiu, X.; et al. Plasmonic nanolaser using epitaxially grown silver film. Science 2012, 337, 450453.

(24) Hou, Y.; Renwick, P.; Liu, B.; Bai, J.; Wang, T. Room temperature plasmonic lasing in a continuous wave operation mode from an InGaN/GaN single nanorod with a low threshold. Sci. Rep. 2014, 4, 5014.

(25) Lu, Y.-J.; Wang, C.-Y.; Kim, J.; Chen, H.-Y.; Lu, M.-Y.; Chen, Y.-C.; Chang, W.-H.; Chen, L.-J.; Stockman, M. I.; Shih, C.-K.; et al. All-color plasmonic nanolasers with ultralow thresholds: autotuning mechanism for single-mode lasing. Nano Lett. 2014, 14, 4381-4388.

(26) Oulton, R. F.; Sorger, V. J.; Zentgraf, T.; Ma, R.-M.; Gladden, C.; Dai, L.; Bartal, G.; Zhang, X. Plasmon lasers at deep subwavelength scale. Nature 2009, 461, 629-632.

(27) Ho, J.; Tatebayashi, J.; Sergent, S.; Fong, C. F.; Iwamoto, S.; Arakawa, Y. Low-Threshold near-Infrared GaAs-AlGaAs Core-Shell Nanowire Plasmon Laser. ACS Photonics 2015, 165-171.

(28) Ho, V. X.; Al Tahtamouni, T. M.; Jiang, H. X.; Lin, J. Y.; Zavada, J. M.; Vinh, N. Q. Room-Temperature Lasing Action in GaN Quantum Wells in the Infrared $1.5 \mu \mathrm{m}$ Region. ACS Photonics 2018, 5, 1303-1309.

(29) Suh, J. Y.; Kim, C. H.; Zhou, W.; Huntington, M. D.; Co, D. T.; Wasielewski, M. R.; Odom, T. W. Plasmonic Bowtie Nanolaser Arrays. Nano Lett. 2012, 12, 5769-5774.

(30) Zhou, W.; Dridi, M.; Suh, J. Y.; Kim, C. H.; Co, D. T.; Wasielewski, M. R.; Schatz, G. C.; Odom, T. W. Lasing action in strongly coupled plasmonic nanocavity arrays. Nat. Nanotechnol. 2013, 8, 506-511.

(31) Dridi, M.; Schatz, G. C. Lasing action in periodic arrays of nanoparticles. J. Opt. Soc. Am. B 2015, 32, 818-823.

(32) Yang, A.; Hoang, T. B.; Dridi, M.; Deeb, C.; Mikkelsen, M. H.; Schatz, G. C.; Odom, T. W. Real-time tunable lasing from plasmonic nanocavity arrays. Nat. Commun. 2015, 6, 6939.

(33) Plum, E.; Fedotov, V. A.; Kuo, P.; Tsai, D. P.; Zheludev, N. I. Towards the lasing spaser: controlling metamaterial optical response with semiconductor quantum dots. Opt. Express 2009, 17, 85488551.

(34) Meng, X.; Kildishev, A. V.; Fujita, K.; Tanaka, K.; Shalaev, V. M. Wavelength-Tunable Spasing in the Visible. Nano Lett. 2013, 13, 4106-4112.

(35) Schokker, A. H.; Koenderink, A. F. Lasing at the band edges of plasmonic lattices. Phys. Rev. B 2014, 90, 155452.

(36) Nagra, A. S.; York, R. A. FDTD Analysis of wave propagation in nonlinear absorbing and gain media. In IEEE Transactions on Antennas and Propagation; IEEE: 1998, 334.

(37) Siegman, A. E. LASERS; University Science Books: California, 1986.

(38) Chen, X.; Bhola, B.; Huang, Y.; Ho, S. T. Multi-level multithermal-electron FDTD simulation of plasmonic interaction with semiconducting gain media: applications to plasmonic amplifiers and nano-lasers. Opt. Express 2010, 18, 17220-17238.

(39) Taflove, A.; Hagness, S. C. Computational Electrodynamics the Finite-Difference Timedomain Method; Artech House: 2005.

(40) Gvozdic, B. D.; Djurdjevic, D. Z. Performance advantages of CPML over UPML absorbing boundary conditions in FDTD algorithm. J. Electr. Eng. 2017, 68, 47-53.

(41) Vial, A.; Grimault, A.-S.; Macías, D.; Barchiesi, D.; de la Chapelle, M. L. Improved analytical fit of gold dispersion: Application to the modeling of extinction spectra with a finite-difference timedomain method. Phys. Rev. B 2005, 71, No. 085416. 
(42) Zou, S.; Janel, N.; Schatz, G. C. Silver nanoparticle array structures that produce remarkably narrow plasmon lineshapes. $J$. Chem. Phys. 2004, 120, 10871-10875.

(43) Hicks, E. M.; Zou, S.; Schatz, G. C.; Spears, K. G.; Van Duyne, R. P.; Gunnarsson, L.; Rindzevicius, T.; Kasemo, B.; Käll, M. Controlling plasmon line shapes through diffractive coupling in linear arrays of cylindrical nanoparticles fabricated by electron beam lithography. Nano Lett. 2005, 5, 1065-1070.

(44) Zhao, L.; Kelly, K. L.; Schatz, G. C. The Extinction Spectra of Silver Nanoparticle Arrays: Influence of Array Structure on Plasmon Resonance Wavelength and Width. J. Phys. Chem. B 2003, 107, 73437350.

(45) Pinchuk, A. O.; Schatz, G. C. Nanoparticle optical properties: Far- and near-field electrodynamic coupling in a chain of silver spherical nanoparticles. Mater. Sci. Eng., B 2008, 149, 251-258. 